Supporting Information

\title{
Facile and Novel eco-friendly Poly(vinyl alcohol) nanofilters using the photocatalytic property of Titanium dioxide
}

Ji Hyun Yeo ${ }^{\mathrm{a}, 1}$, Myounguk Kim ${ }^{\mathrm{b}, 1}$, Hakjun Lee ${ }^{\mathrm{a}}$, Jihyun Cho ${ }^{\mathrm{a}}$, and Jongshin Parka, c,*

a. Department of Biosystems \& Biomaterials Science and Engineering Seoul National University, Seoul 08826, Republic of Korea

b. Fibrous Ceramics \& Aerospace Materials Center, Korea Institute of Ceramic Engineering and Technology, Jinju 52851, Republic of Korea

c. Research Industry of Agriculture and Life Sciences, Seoul National University, Seoul 08826, Republic of Korea 


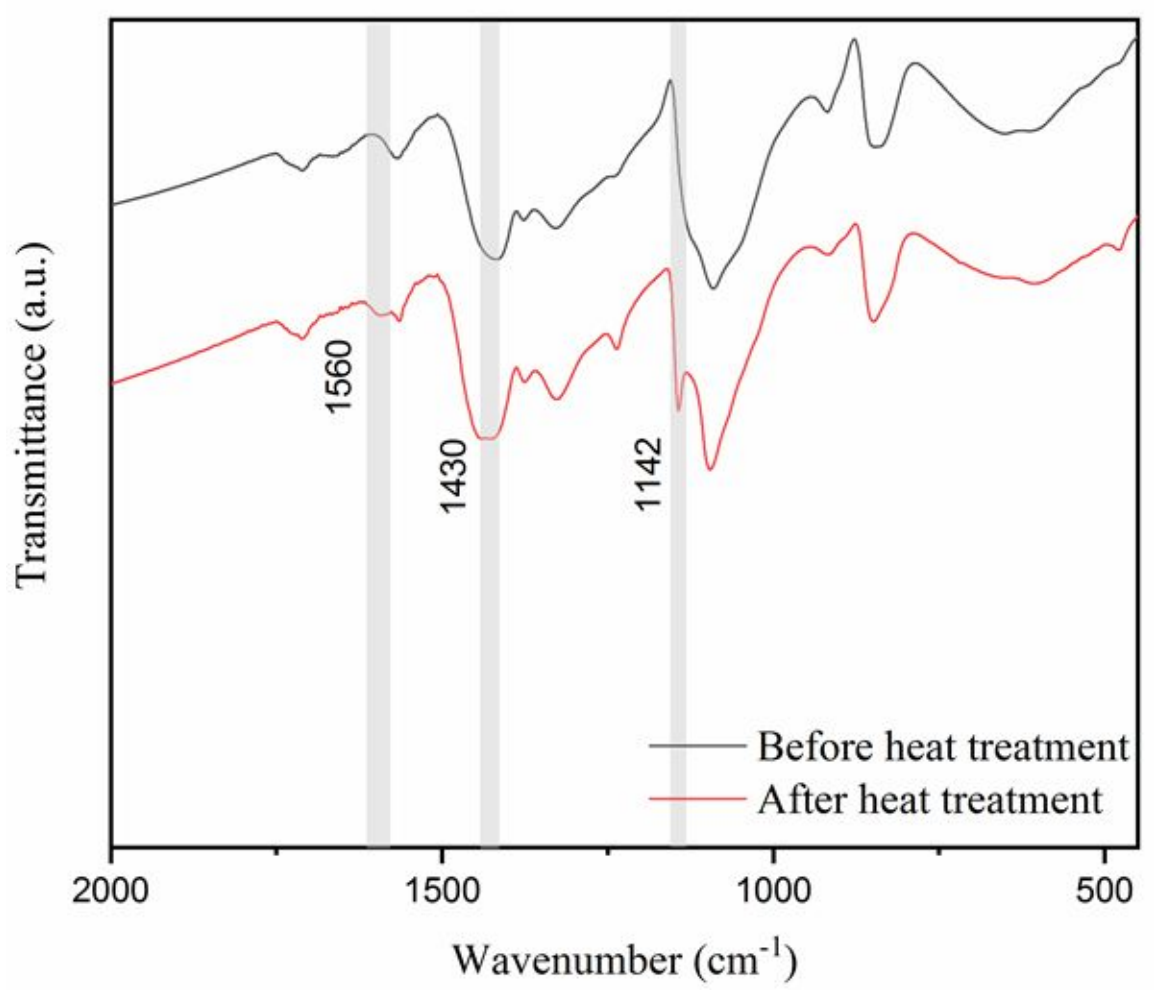

Figure S1. FT-IR spectra of PVA nanofilter before and after heat treatment. 

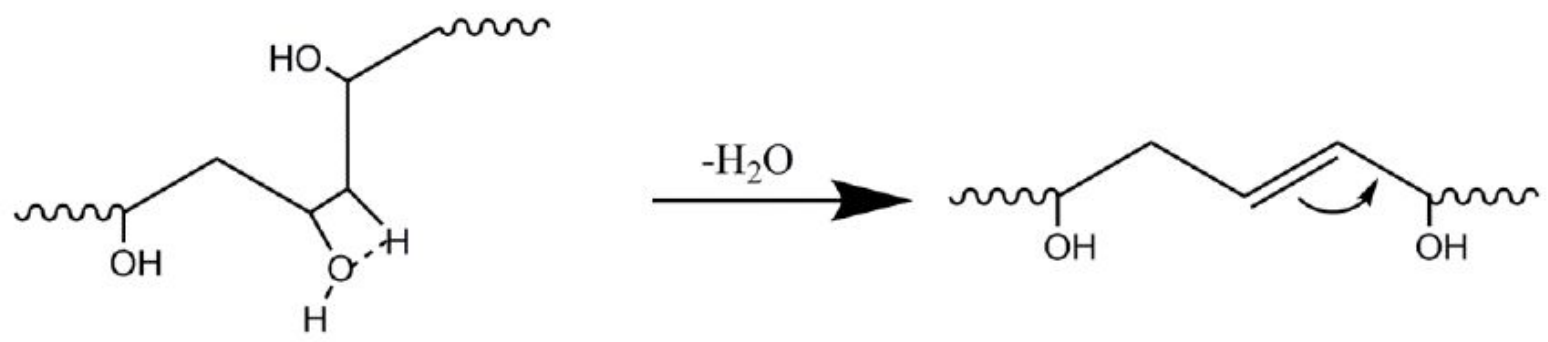

Double bond migration
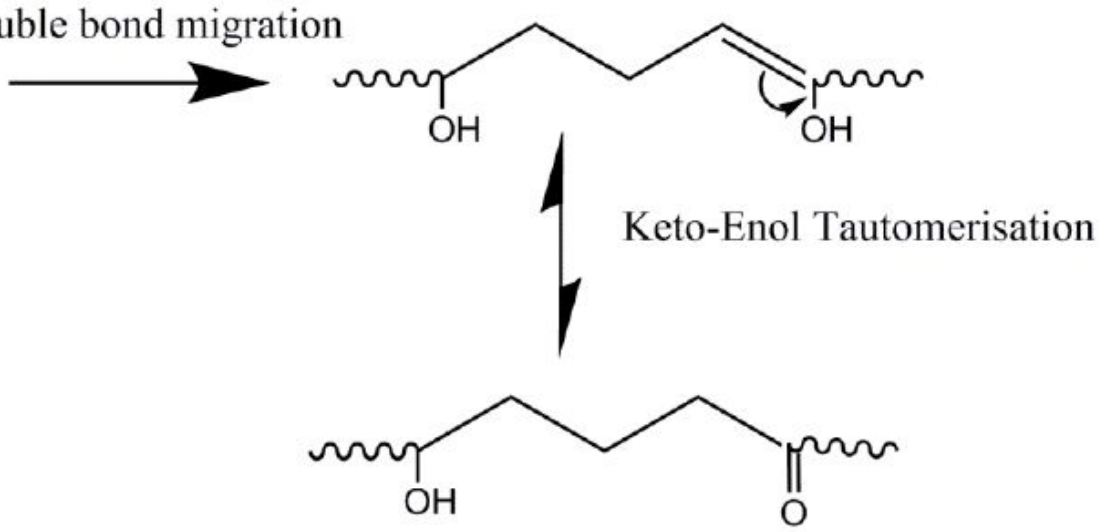

Scheme S1. Keto-enol tautomerization of PVA nanofilter during heat treatment. 
Table S1. Average Filter Thickness of PVA nanofilters

\begin{tabular}{|c|c|}
\hline Time (h) & Average Filter Thickness \\
& (um) \\
\hline 1 & $22 \pm 4.47$ \\
\hline 2 & $32 \pm 8.37$ \\
\hline 3 & $38 \pm 8.37$ \\
\hline 4 & $70 \pm 18.03$ \\
\hline 5 & $126 \pm 23.02$ \\
\hline 6 & $140 \pm 36.74$ \\
\hline 7 & $170 \pm 36.74$ \\
\hline
\end{tabular}




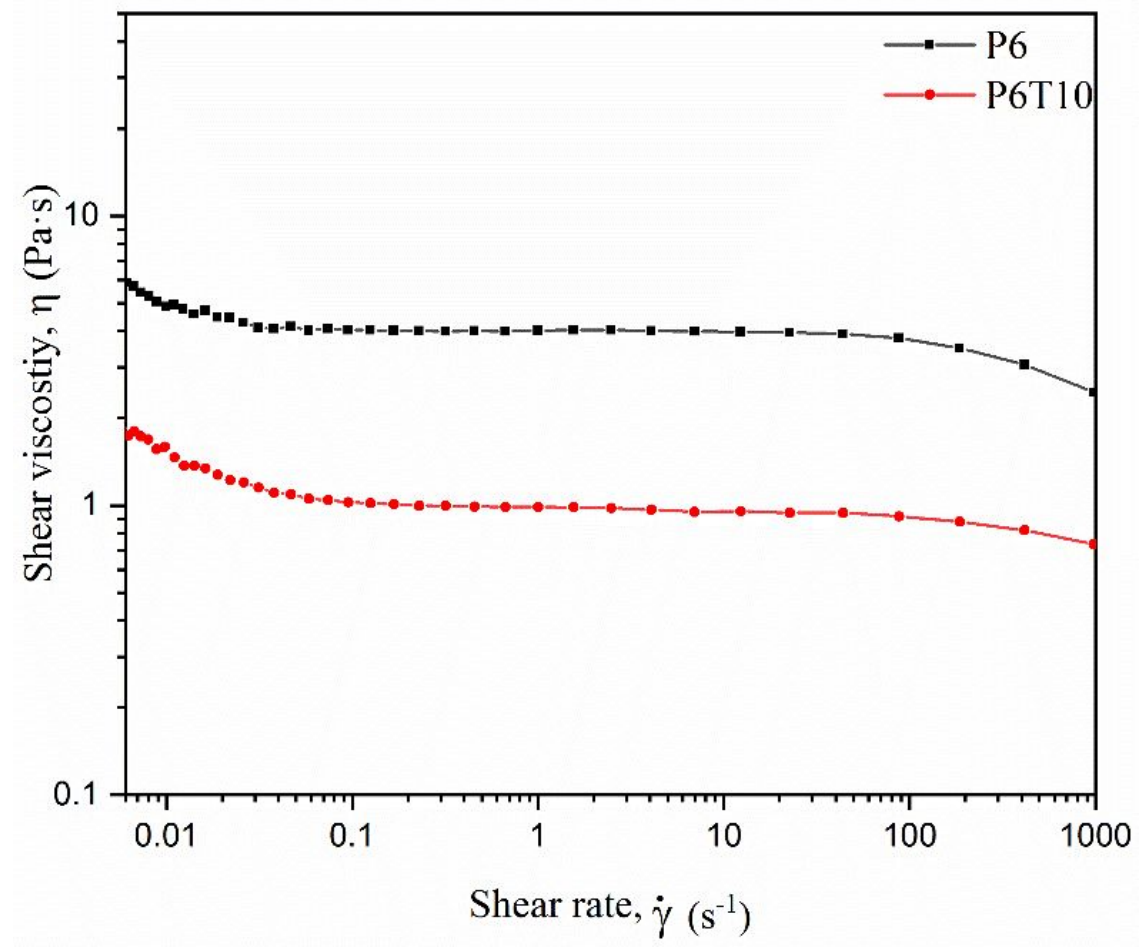

Figure S2. Shear viscosity of electrospinning solutions of P6 and P6T10. 
Table S2. Average fiber diameter of $\mathrm{PVA} / \mathrm{TiO}_{2}$ nanofilters.

\begin{tabular}{|c|c|}
\hline Sample & $\begin{array}{c}\text { Average Fiber Diameter } \\
(\mathrm{nm})\end{array}$ \\
\hline P6 & $584 \pm 167$ \\
\hline P6T2.5 & $412 \pm 88$ \\
\hline P6T5 & $349 \pm 99$ \\
\hline P6T7.5 & $276 \pm 63$ \\
\hline P6T10 & $224 \pm 32$ \\
\hline
\end{tabular}



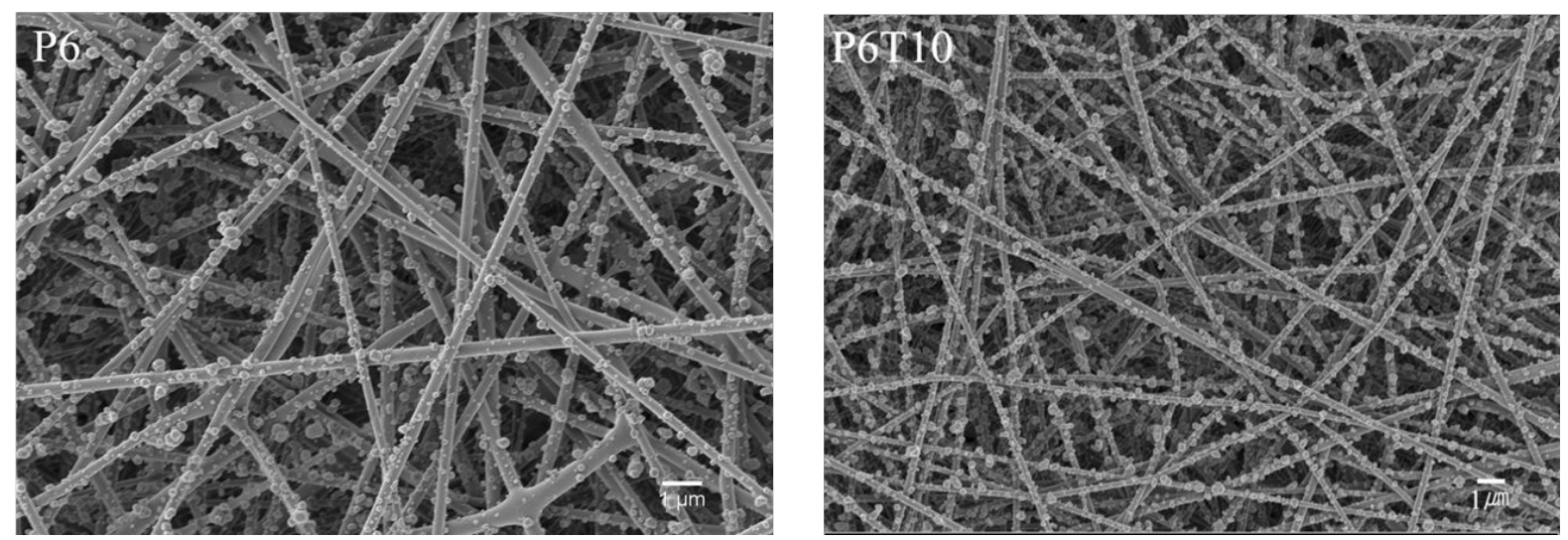

Figure S3. FE-SEM image of P6 and P6T10 nanofilter after the filtration test. 


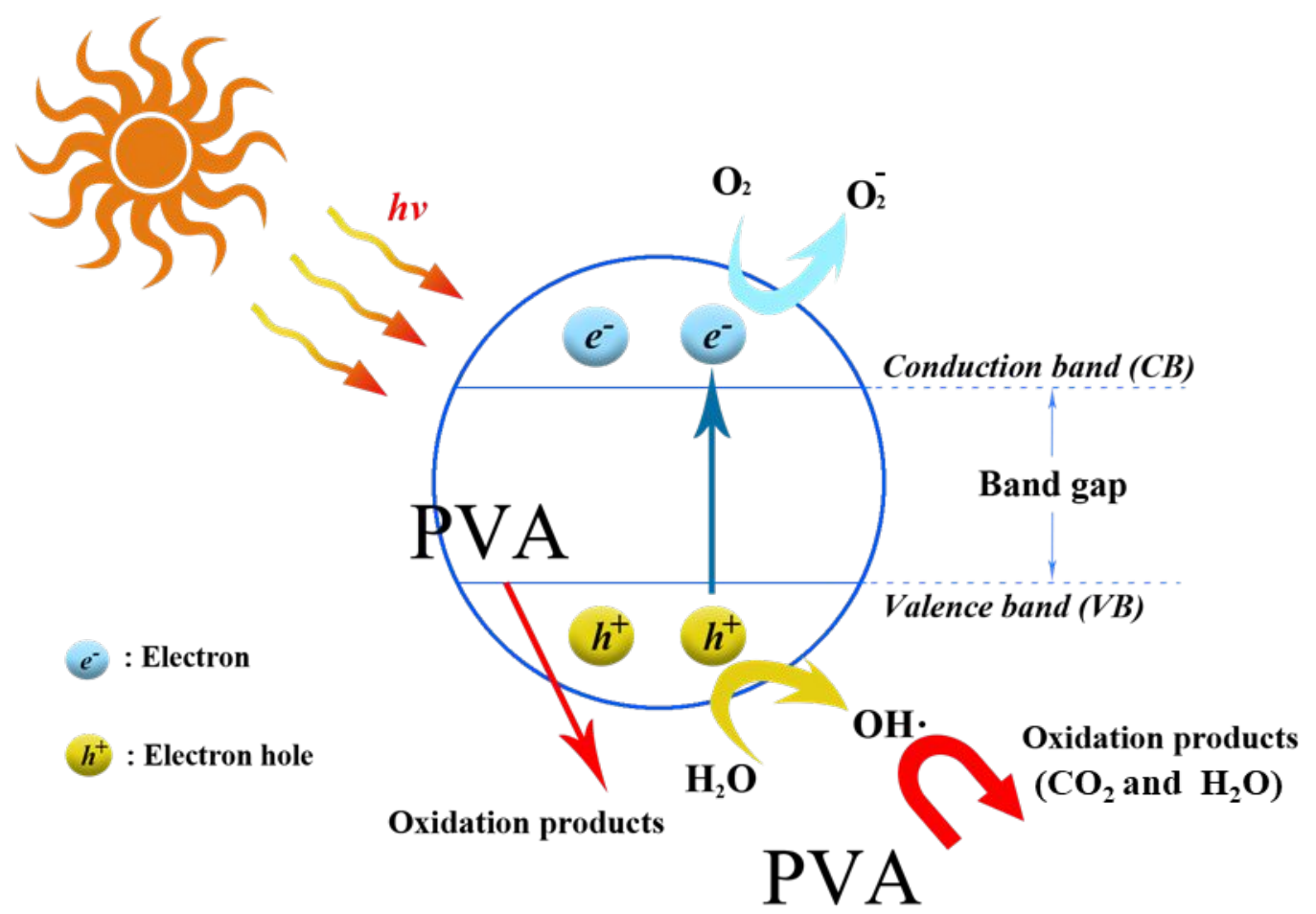

Scheme S2. Photocatalytic decomposition of $\mathrm{PVA} / \mathrm{TiO}_{2}$ nanofilter. 
Table S3. Weight loss of $\mathrm{PVA} / \mathrm{TiO}_{2}$ nanofilters at different UV irradiation times.

\begin{tabular}{|c|c|c|c|c|c|}
\hline \multirow{2}{*}{$\begin{array}{c}\text { UV irradiation } \\
\text { time } \\
(\text { Day) }\end{array}$} & \multicolumn{6}{|c|}{ Weight loss (\%) } \\
\cline { 2 - 7 } & P6 & P6T2.5 & P6T5 & P6T7.5 & P6T10 \\
\hline 3 & - & $5.38 \pm 1.37$ & $7.02 \pm 0.63$ & $14.21 \pm 0.15$ & $19.92 \pm 0.62$ \\
\hline 6 & - & $8.96 \pm 0.10$ & $19.32 \pm 0.63$ & $\begin{array}{c}11.26 \pm \\
11.86\end{array}$ & $68.19 \pm 3.22$ \\
\hline 9 & - & $21.75 \pm 1.07$ & $39.57 \pm 5.44$ & $62.28 \pm 6.86$ & $72.11 \pm 4.44$ \\
\hline
\end{tabular}



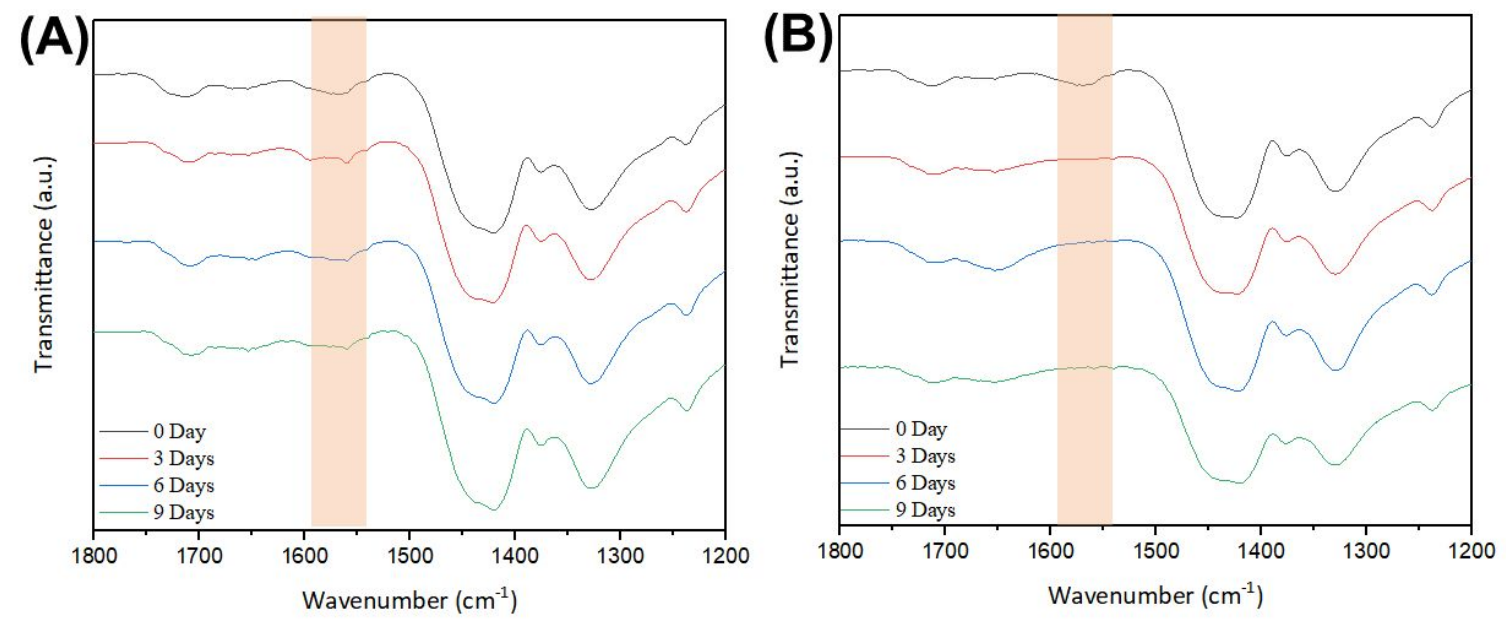

Figure S4. FT-IR spectra in range of $1200-1800 \mathrm{~cm}^{-1}$ for (A) P6 and (B) P6T10. 